Supplement of Biogeosciences, 15, 1203-1216, 2018

https://doi.org/10.5194/bg-15-1203-2018-supplement

(C) Author(s) 2018. This work is distributed under

the Creative Commons Attribution 3.0 License.

(c) (1)

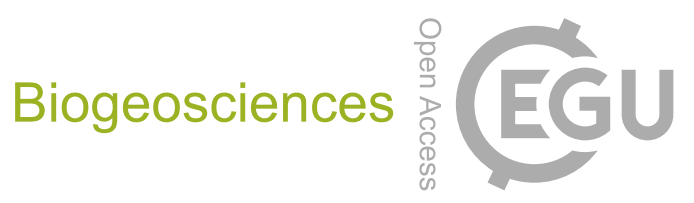

Supplement of

\title{
Catchment tracers reveal discharge, recharge and sources of groundwater-borne pollutants in a novel lake modelling approach
}

Emil Kristensen et al.

Correspondence to: Emil Kristensen (emil.kristensen@bio.ku.com)

The copyright of individual parts of the supplement might differ from the CC BY 3.0 License. 

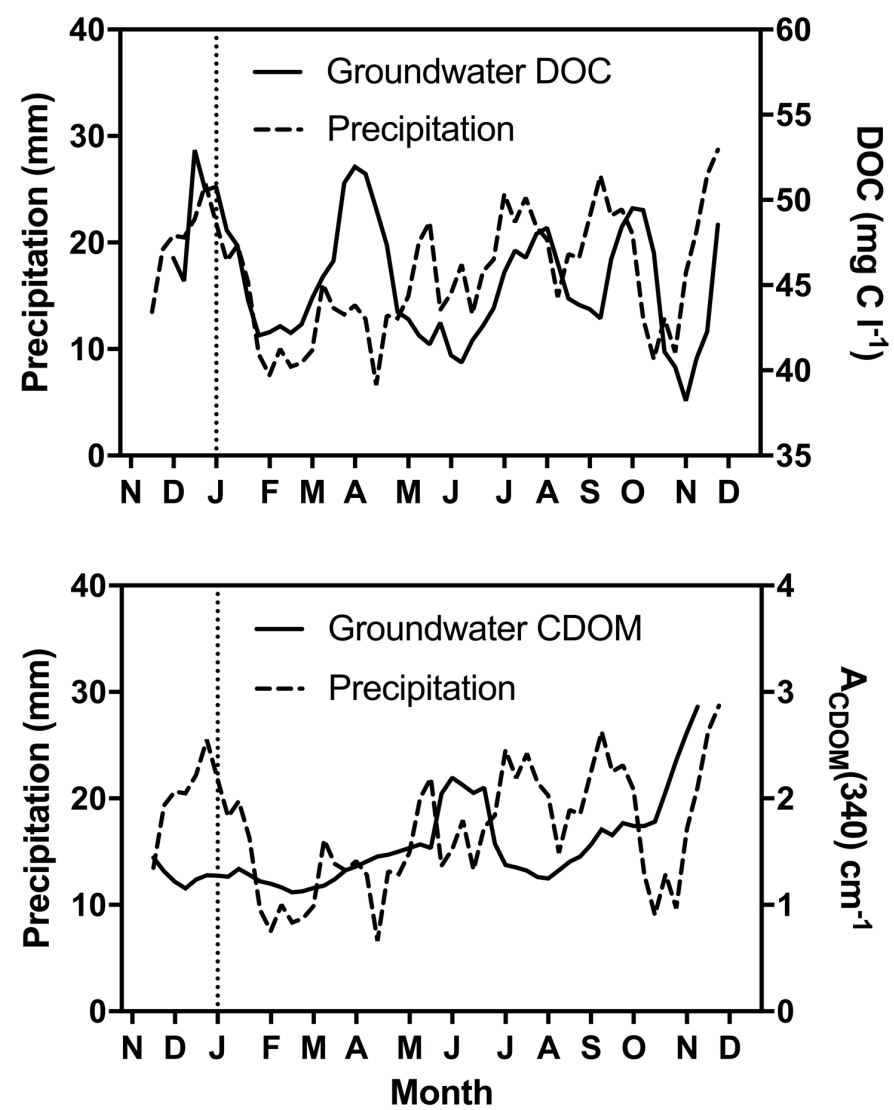

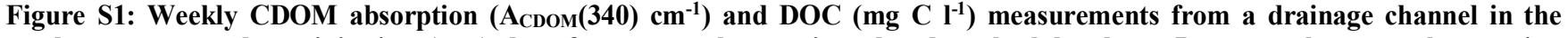
catchment area and precipitation $(\mathrm{mm})$ data from a weather station placed at the lake shore. Data are shown as the running average of four weeks. 


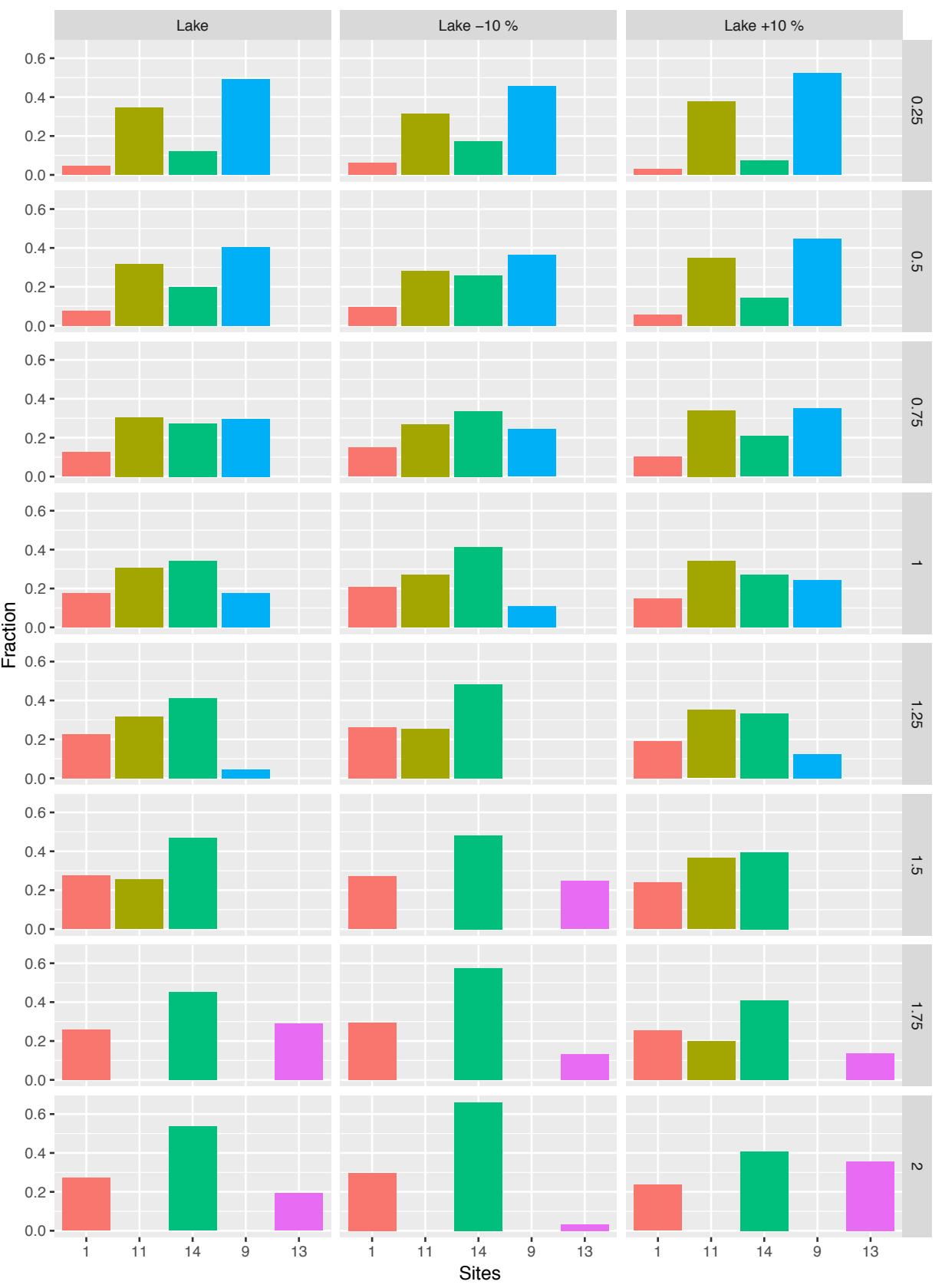

Figure S2: Bar plot showing the results from the sensitivity analysis derived from the CATS model. The top $x$-axis shows the different concentrations used for the lake e.g. no change (Lake), $10 \%$ reduction (Lake $-10 \%)$ and $10 \%$ increment (Lake +10 . The left $y$-axis denotes the estimated fractions of inflowing groundwater originating from the groundwater well sites (bottom $x$-axis, 5 only sites delivering more than 0.01 are shown) and the second $y$-axis denotes the corresponding WRT. In general, a smaller variance between sites and discharge fractions is seen up to a WRT of 1.25 years. Above this point there is some changes between site 11 and 13. Overall, the same 5 groundwater well sites are isolated which explain the measured lake concentrations. 
Table S1: Tracer concentrations of $\delta^{18} \mathrm{O}(\%)$, dissolved organic carbon (DOC $\mu \mathrm{mol}^{-1}$ ), coloured dissolved organic matter $\left(\mathrm{A}_{\text {СDом }}(340) \mathrm{cm}^{-1}\right.$ ), total dissolved phosphorus (TDP $\mu \mathrm{g} \mathrm{l}^{\mathrm{l}^{-1}}$ ), total phosphorus (TP $\mu \mathrm{g} \mathrm{l}^{-1}$ ), total dissolved nitrate (TDN $\mu \mathrm{g} \mathrm{l^{-1 }}$ ), total nitrate (TN $\mu \mathrm{g}^{-1}$ ) and maximum fluorescence of component C1-C5 in Raman's units (R.U.) found around the lake (1-30), in the lake $(a, b$ and $c)$ and at a water locked (WL) site. Dissolved nutrients are measured in groundwater while total fractions are found 5 in lake water. For site reference see figure $1 \mathrm{a}$.

\begin{tabular}{|c|c|c|c|c|c|c|c|c|c|c|}
\hline Sites & $\begin{array}{c}\delta^{18} \mathrm{O} \\
\% 0\end{array}$ & $\begin{array}{c}\mathrm{DOC} \\
\mu \mathrm{mol} \mathrm{l}^{-1}\end{array}$ & $\begin{array}{c}\text { CDOM } \\
\mathrm{A}_{\mathrm{CDOM}}(340) \\
\mathrm{cm}^{-1} \\
\end{array}$ & $\begin{array}{c}\mathrm{TDP} / \mathrm{T} \\
\mathrm{P} \\
\mu \mathrm{g} \mathrm{^{-1 }} \\
\end{array}$ & $\begin{array}{c}\text { TDN/T } \\
\mathrm{N} \\
\mu \mathrm{g} \mathrm{l}^{-1} \\
\end{array}$ & $\begin{array}{c}\text { C1 } \\
\text { R.U. }\end{array}$ & $\begin{array}{c}\text { C2 } \\
\text { R.U. }\end{array}$ & $\begin{array}{c}\text { C3 } \\
\text { R.U. }\end{array}$ & $\begin{array}{c}\text { C4 } \\
\text { R.U. }\end{array}$ & $\begin{array}{c}\text { C5 } \\
\text { R.U. }\end{array}$ \\
\hline Lake (a) & & 952 & 0.40 & 36 & 708 & 7.8 & 3.1 & 2.1 & 1.1 & 0.3 \\
\hline Lake (b) & -4.6 & 1117 & 0.41 & 28 & 662 & 7.8 & 3.1 & 2.2 & 1.1 & 0.3 \\
\hline Lake (c) & -4.6 & 1105 & 0.40 & 29 & 652 & 7.8 & 3.1 & 2.2 & 1.1 & 0.3 \\
\hline 1 & -7.2 & 3115 & 1.29 & 42 & 994 & 14.9 & 6.8 & 4.3 & 3.1 & 0.7 \\
\hline 2 & -9.2 & 5032 & 1.53 & 105 & 1051 & 21.6 & 9.8 & 7.8 & 3.8 & 1.0 \\
\hline 3 & -9.0 & 7782 & 2.04 & 102 & 1148 & 32.7 & 14.2 & 11.6 & 5.1 & 1.3 \\
\hline 4 & -8.6 & 10467 & 2.50 & 167 & 1671 & 44.3 & 18.0 & 15.7 & 5.1 & 1.1 \\
\hline 5 & -9.4 & 5735 & 1.59 & 90 & 1031 & 28.7 & 12.4 & 10.9 & 3.7 & 0.7 \\
\hline 6 & -8.2 & 8791 & 3.11 & 134 & 1477 & 36.3 & 16.3 & 12.6 & 6.2 & 1.5 \\
\hline 7 & -8.2 & 9298 & 2.94 & 124 & 1419 & 47.1 & 21.6 & 15.8 & 7.6 & 1.9 \\
\hline 8 & -7.3 & 4943 & 1.48 & 64 & 1250 & 26.7 & 14.3 & 11.2 & 5.2 & 2.9 \\
\hline 9 & -6.6 & 1721 & 0.31 & 54 & 405 & 7.0 & 3.4 & 2.6 & 1.1 & 0.1 \\
\hline 10 & -7.3 & 4483 & 0.58 & 17 & 856 & 20.8 & 8.9 & 9.1 & 2.3 & 0.3 \\
\hline 11 & -6.9 & 2504 & 0.32 & 9 & 764 & 6.9 & 3.1 & 2.9 & 0.9 & 0.1 \\
\hline 12 & -6.6 & 4229 & 0.55 & 18 & 857 & 13.7 & 6.2 & 5.9 & 2.0 & 0.2 \\
\hline 13 & -6.8 & 3848 & 0.64 & 34 & 1285 & 13.8 & 6.2 & 6.0 & 2.0 & 0.4 \\
\hline 14 & -7.0 & 5868 & 0.87 & 124 & 2198 & 13.9 & 5.7 & 6.2 & 1.2 & 0.3 \\
\hline 15 & -6.7 & 10656 & 2.41 & 59 & 2309 & 41.1 & 20.7 & 16.1 & 6.1 & 1.8 \\
\hline 16 & -4.3 & 2674 & 0.26 & 4 & 325 & 7.6 & 3.3 & 2.1 & 1.6 & 0.4 \\
\hline 17 & -5.2 & 3115 & 0.24 & 12 & 589 & 7.5 & 3.3 & 2.1 & 1.8 & 0.4 \\
\hline 18 & -4.8 & 5032 & 0.24 & 15 & 726 & 7.6 & 3.2 & 2.2 & 1.7 & 0.4 \\
\hline 19 & -4.7 & 7782 & 0.27 & 1 & 592 & 7.6 & 3.3 & 2.1 & 1.5 & 0.6 \\
\hline 20 & -4.6 & 10467 & 0.32 & 7 & 522 & 7.6 & 3.2 & 2.2 & 1.2 & 0.5 \\
\hline 21 & -4.5 & 5735 & 0.31 & 10 & 657 & 7.6 & 3.2 & 2.3 & 1.3 & 0.6 \\
\hline 22 & -4.7 & 8791 & 0.34 & 10 & 656 & 7.6 & 3.3 & 2.2 & 1.4 & 0.5 \\
\hline 23 & -5.3 & 9298 & 0.34 & 30 & 667 & 7.6 & 3.3 & 2.2 & 1.3 & 0.3 \\
\hline 24 & -5.1 & 4943 & 0.52 & 26 & 709 & 7.7 & 3.0 & 2.4 & 1.1 & 0.3 \\
\hline 25 & -4.6 & 1721 & 0.80 & 30 & 1016 & 15.0 & 5.9 & 5.1 & 1.8 & 0.5 \\
\hline 26 & -4.6 & 4483 & 0.32 & 66 & 436 & 7.7 & 3.1 & 2.2 & 1.3 & 0.3 \\
\hline 27 & -44 & 2504 & 0.30 & 38 & 511 & 7.6 & 3.3 & 2.0 & 1.6 & 0.3 \\
\hline 28 & -5.1 & 4229 & 0.90 & 65 & 859 & 15.0 & 6.2 & 4.8 & 2.1 & 0.5 \\
\hline 29 & -4.3 & 3848 & 1.21 & 81 & 775 & 18.5 & 8.4 & 5.7 & 3.7 & 1.0 \\
\hline 30 & -6.8 & 5868 & 1.09 & 59 & 788 & 17.4 & 9.3 & 6.0 & 4.3 & 0.9 \\
\hline WL & -6.7 & 4276 & 2.6 & 193 & 2388 & 41.7 & 16.4 & 19.5 & 3.5 & 2.6 \\
\hline
\end{tabular}

